\title{
Revendication et expression de liberté dans les dramaturgies africaines postcoloniales chez Sony Labou Tansi et Bottey Zadi Zaourou
}

Fétigué COULIBALY, École Normale Supérieure d'Abidjan (Côte d'Ivoire)

Revendiquer, acte aussi conscient que volontaire consistant à demander avec force, à réclamer et à exiger ce à quoi l'on a absolument droit, devient l'une des préoccupations essentielles des peuples d'Afrique au lendemain des indépendances. Le vent de la revendication et de la libération politique qui, en Afrique, a abouti aux « soleils des indépendances » cède la place, une fois les indépendances acquises, à une autre revendication, cette fois, relative à l'émancipation de la littérature en général et du théâtre africain en particulier. En effet, si tant est que, selon Victor Hugo, « la liberté littéraire est fille de la liberté politique » et qu' « à peuple nouveau, art nouveau » (Liourré 51), c'est une «indispensabilité » pour l'Afrique qui a recouvré sa liberté politique d'accoucher d'un théâtre authentiquement africain afin « de se redéfinir, de se retrouver, de rétablir le contact avec [elle]-même, de rétablir la continuité psychologique » (Kotchy et Kesteloot 144) indispensable pour tout peuple conscient de son aliénation. Cette liberté dramaturgique tant souhaitée consiste en une renaissance littéraire que Jean-Marie Adiaffi explique ainsi : « la renaissance littéraire en ce qui nous concerne est la finalité ultime de nos recherches. Partir de la littérature africaine pour innover, trouver de nouvelles formes » (20). Lui faisant écho, Boubacar Siné ajoute que «l'émancipation de notre littérature, son élévation à la majorité et à la dignité d'une littérature nationale, nous devons la payer au prix d'une rupture déchirante avec le modèle littéraire dominant d'importation coloniale» (cité dans Evelyne Lavergne 15). Ce faisant, l'histoire de la création théâtrale en Afrique, depuis les indépendances jusqu'à nos jours, s'inscrit irréductiblement dans un processus continu d'une véritable fabrique de l'identité, de l'authenticité africaine, voire de l'africanité. Comment les auteurs africains expriment-ils leur liberté dramaturgique ? La présente étude se propose d'élucider les innovations qui manifestent la liberté dramaturgique et consacrent l'émergence d'une esthétique d'essence africaine.

\section{L'indépendance dramaturgique chez les auteurs africains de la première génération}

Le théâtre africain moderne, c'est-à-dire écrit et d'expression française, tire ses origines

profondes des écoles coloniales, notamment l'École Primaire Supérieure de Bingerville en Côte 
d'Ivoire et l'École Normale William-Ponty de Gorée au Sénégal. Dans ces institutions coloniales, la jeunesse scolaire africaine fut initiée tant à l'écriture qu'à la pratique théâtrale à l'occidentale. À ce propos, Amon d'Aby écrit :

Un jeudi de l'année 1932, à l'École Primaire Supérieure de Bingerville, les élèves qui venaient de laver leur linge se regroupaient au réfectoire, sorte de hangar en bois soutenu par des piliers en ciment. Deux jeunes de deuxième année entreprirent d'amuser leurs camarades. Ils le firent spontanément. L'un, Édouard Aka Bilé, aujourd'hui décédé, s'habilla sommairement en agent de police. L'autre Robert Animan, jetant sur son épaule une vieille couverture, se transforma en chef de village. Tous deux improvisèrent sur une affaire d'impôt une discussion de quelques minutes qui furent vivement applaudie. M. Charles Béart, Directeur de l'École et sa femme qui, de leur fenêtre, avaient assisté à cette plaisanterie sortirent pour en féliciter les auteurs, et décidèrent que désormais les mercredi et samedi soir seraient consacrés à des distractions de ce genre. Une scène en terre battue fut aussitôt construite dans la cour. Dès lors, régulièrement des épisodes amusants de la vie indigène furent représentés sur cette scène rudimentaire. Les acteurs improvisaient sur un scénario, avec un brio tel que, dès la fin de 1993, des représentations purent être données devant un public européen, notamment à l'occasion de la foire-exposition d'Abidjan en janvier 1934. (cité dans Traoré, « Ecole », 39)

Faisant écho à Aby, Bakary Traoré ajoute qu' « à la fin de l'année scolaire 1932-1933, une pièce composée par les élèves dahoméens, Bayot et Béhanzin, prit place à côté d'une farce de Molière. C'est la première manifestation connue du théâtre africain d'expression française » (39). Ce théâtre est, certes, africain mais il est conçu sous le contrôle strict et minutieux du colon si bien qu'il y était formellement proscrit toute prise de position vis-à-vis des problèmes du moment. La thématique de prédilection de ce théâtre était simplement d'ordre socioculturel et inscrite sur la trajectoire moliéresque. Autrement dit, il plongeait ses racines dans les arcanes des folklores africains en dénonçant des aspects jugés négatifs, en particulier le problème de la succession dans la chefferie traditionnelle tout comme dans la royauté, la question de l'héritage, du rejet de certains enfants comme le dixième dans la culture agni de Côte d'Ivoire, etc.

L'avènement des indépendances ouvre une ère nouvelle pour le théâtre africain moderne. À la faveur des indépendances, l'épée de Damoclès qui, à l'époque du colonialisme triomphant, était suspendue au-dessus de la tête des jeunes dramaturges africains, interdisant et condamnant avec la dernière énergie toute prise de position vis-à-vis des problèmes du moment, disparait. Cette situation cède la place à un espace et un temps de liberté d'expression qui permettra aux nouveaux dramaturges africains de s'exprimer pleinement, en toute liberté, et de révéler sans ambages et 
sans complaisance les problèmes auxquels leurs peuples se trouvent confrontés dans leur nouvel univers social et dans leur vie de tous les jours. En effet, au lendemain des indépendances politiques, le théâtre africain moderne prend son envol, c'est-à-dire connaît un essor sans précédent, par une significative rénovation et une adaptation de sa thématique. Cette dernière est désormais adaptée aux nouveaux contextes sociaux issus des indépendances. Elle n'évoque et ne débatte que des questions proprement africaines. En d'autres termes, la toile de fond principale des fictions théâtrales africaines au lendemain des indépendances gravite essentiellement autour de la problématique du pouvoir politique, la structuration sociale, les orientations idéologiques, le conflit vécu et actuel entre la tradition africaine et la modernité, sans oublier l'histoire. Dans cette thématique novatrice et fécondante, le thème de la politique demeure prépondérant. C'est pourquoi, Aimé Césaire, l'une des icônes de ce théâtre, avoue à juste titre, « mon théâtre est surtout politique parce que les problèmes majeurs en Afrique sont des problèmes politiques » (Kotchy et Kesteloot 121). Comme «l'art reflète la culture dont il est issu » (Sido 94), le théâtre, une des manifestations les plus vivantes de la culture, révèle avec assez de pertinence et d'efficacité les caractéristiques essentielles de la société. Les dramaturges plongent dans la réalité politique africaine en vue de présenter au public des thèmes qui répondent à ses préoccupations fondamentales si bien que l'engagement devient la nouvelle orientation et la tendance de prédilection du théâtre africain au lendemain des indépendances.

Cette période de l'histoire marque une ère nouvelle pour l'Afrique qui a vraiment besoin de comprendre les enjeux et les défis à relever, de se ressaisir et se reconstruire :

Avec l'accès à l'indépendance des pays africains, écrit Aimé Césaire, nous sommes entrés dans le moment de la responsabilité. Les Noirs désormais doivent faire leur histoire. Et l'histoire des Noirs sera vraiment ce qu'ils en auront fait. Ça me paraît assez naturel qu'au moment où on accède à la responsabilité, on jette un regard en arrière. On s'interroge soi-même, on essaie de comprendre, or dans le siècle où nous sommes, la poésie est un langage qui nous paraît plus ou moins ésotérique. Il faut parler clair, parler net, pour faire passer le message. Il me semble que le théâtre peut s'y prêter et s'y prête bien. (Kotchy et Kesteloot 120)

Dans cette perspective, la rénovation thématique du théâtre et son adaptation aux questions spécifiquement africaines relèvent d'une volonté des dramaturges de l'authentifier, de le nationaliser ou de l'africaniser et en faire un art original qui prend la forme et le sens d'une affirmation de la personnalité et de l'identité des peuples d'Afrique. Cette initiative est le fait de dramaturges africains formant ce qu'il convient d'appeler la première génération. En effet, pour 
des peuples qui ont connu la colonisation, l'acquisition d'une littérature en général ou d'un théâtre en particulier, s'avère absolument indispensable car le théâtre, avouons-le, est un univers d'images. Et l'image, on le sait, est à la fois signe et signification, c'est-à-dire message et communion, mais aussi source de culture et pédagogie de prise de conscience. Le théâtre est, de toutes les vieilles civilisations, notamment la civilisation négro-africaine, l'instrument le plus efficace de la transformation sociale; il ne se contente pas de former le gout, d'aiguiser la sensibilité mais il éduque l'homme et l'oriente vers la voie de son salut. Il est, pour le monde africain, cet art qui informe, forme l'homme ainsi que la société tout en le plongeant dans cet univers civilisationnel où le passé, le présent et l'avenir se confondent, c'est-à-dire où le passé devient présent et le présent avenir, ce qui en fait une reconstitution historique de la vie des ancêtres, un fondement de culture qui « traduit la totalité des manifestations de la vie » (Traoré 54) africaine, mais aussi un moyen de sensibilisation où «les leçons politiques et sociales jouées frappent plus que des slogans et des discours » (Kotchy 11). Il est un révélateur et une force mobilisatrice qui fonctionne comme un jeu d'enjeu dans la mesure où, comme le souligne Guillaume Oyono, «ce n'est qu'en le divertissant réellement qu'on peut espérer amener le public à prendre conscience de certains aspects de notre culture ou de notre vie sociale » (Kotchy 11). En effet, le dramaturge «ne développe pas des idées abstraites, mais crée une réalité imaginaire et les possibilités de cette création ne dépendent pas en premier lieu de ses intuitions mais de la réalité sociale au sein de laquelle il vit et des cadres mentaux qu'elle a contribués à élaborer » (Goldman 239).

Le texte littéraire se conçoit aussi comme un ensemble de confluences historiques, culturelles et idéologiques observées dans une vie quotidienne mais fictionnalisées dans l'espace textuel. Il est une unité d'interférences conscientes ou inconscientes. La conscience de tout dramaturge s'abreuve à la source de faits avérés que renferme et refoule son inconscient qui éclate pour libérer les souvenirs enfouis au moment de l'acte d'écriture. Les dramaturges africains ont pour intention, au lendemain des indépendances, d'une part, de prouver au Maître (occidental) que l'élève (africain) a enfin grandi, gagné en sagesse et est doté de potentialités intellectuelles lui permettant d'accoucher de belles et grandes choses autant que le Maître, voire même plus que lui et, d'autre part, de donner à leurs peuples leur vraie personnalité, leur identité réelle. À ce propos, Césaire écrit : «J'aimerais réactualiser la culture noire pour en assumer la permanence pour qu'elle devienne une culture qui contribuerait à l'édification d'un ordre nouveau, d'un ordre 
révolutionnaire où la personnalité africaine pourrait s'épanouir » (cité dans Kotchy et Kestellot 124). Les dramaturges noirs dits de la première génération écrivent le théâtre africain tout en se conformant strictement aux modèles de création théâtrale hérités de l'Occident. Ils s'inscrivent dans un conformisme quasi absolu ou se contentent simplement de calquer les canons dramaturgiques de l'Occident, qu'il s'agisse de la structure externe, c'est-à-dire des grandes unités dramatiques de découpage du texte de théâtre, qu'il s'agisse aussi de la structure interne ou le traitement esthétique de la fable dramatique. Toutes leurs compositions dramaturgiques sont calquées sur le modèle dramaturgique occidental et vouent un culte singulier au français, langue du Maître.

Notons qu'au lendemain des indépendances, la situation linguistique de l'Afrique s'avère catastrophique, car le colon a délibérément désagrégé les langues africaines et imposé sa propre langue, la langue française, au point que le français devient la langue officielle de tous les pays africains colonisés par la France. Ce faisant, pour les dramaturges africains de la première génération, la langue française cesse d'être un simple moyen d'expression et de communication pour devenir « un objet de vénération, une fin en soi » (Makward 31) si bien que l'obsession de la correction grammaticale et syntaxique ne cessait de les hanter davantage. Le français est la langue du colon et celle des dramaturges africains, des ex-colonisés. Dans cette logique, il est tout à fait évident que, devant la langue française, ils manifestent, de façon indépendante de leur volonté, une forme de paralysie dans leur volonté créatrice avec la langue française. Cette paralysie s'est très tôt transformée chez Léopold Sédar Senghor en éloges formulés à l'endroit de la langue du colon. En effet, répondant à une question rhétorique bien sienne « pourquoi, dès lors, écrivez-vous en français ? », l'écrivain sénégalais dit : «parce que nous sommes des métis culturels, parce que si nous sentons en nègres, nous nous exprimons en français, parce que le français est une langue à vocation universelle, que notre message s'adresse aussi aux Français de France et aux autres hommes, parce que le français est une langue de gentillesse et d'honnêteté » (Makward 29). Les dramaturges africains de la première génération se sont parfaitement accommodés avec la langue française en en faisant un usage de grande érudition si bien qu' « aujourd'hui encore des écrivains français considèrent qu'il n'est de belle langue que de Paris. La langue française écrite à Paris doit, selon eux, être l'étalon or de la langue française » (34). L'anéantissement systématique des cultures et surtout des langues africaines ne peut qu'aboutir à une telle situation linguistique déplorable et même tragique. Pendant que le colon blanc les a définitivement plongées dans le 
monde de l'oubli et du néant, il a implanté et imposé la langue française qui ne cesse de s'épanouir pleinement, de prendre de l'ampleur et de s'enrichir davantage. Ainsi, la langue française envahit entièrement et égoïstement l'espace africain étouffant même toute autre langue. En somme, à la remorque du modèle de création théâtrale occidental, l'expression dramatique africaine de la première génération est singulièrement imitative et conformiste.

\section{L'indépendance dramaturgique chez les dramaturges africains de la deuxième génération}

Autour des années quatre-vingt, une nouvelle génération de dramaturges africains voit le jour. Ces dramaturges dits de la deuxième génération se révèlent particulièrement frondeurs et réfractaires à toute forme d'imitation et de conformisme. Ayant radicalement rompu les amarres avec l'hégémonie culturelle occidentale, ils se sont frayé une voie nouvelle dans l'écriture dramaturgique parce que leur volonté est d'affranchir le théâtre africain de l'impérialisme du canon dramaturgique d'importation occidentale, de l'écrire autrement et surtout de lui donner un souffle tout nouveau et tout différent. Leurs compositions dramaturgiques ou pièces théâtrales, par les divers et différents principes de construction dont elles font preuve, donnent matière à l'identification de nouvelles écritures dramatiques, devenant ainsi des œuvres dépositaires d'innovations autant textuelles que scéniques. En d'autres termes, elles s'écartent des conventions du genre et inaugurent un réel renouvellement des formes dramatiques. Leurs écritures deviennent des écritures du bouleversement, de la subversion et de la révolution. Ainsi, ils s'affirment en s'opposant à leurs prédécesseurs de la première génération qui se sont contentés de se confiner dans l'imitation et le conformisme. Considérant ce conformisme comme la source de leur aliénation, de leur contrôle intellectuel et de la paralysie de leur génie créateur et instituant l'anticonformisme, la transgression ou la rupture comme principe et critère intangible de création théâtrale, les dramaturges de la deuxième génération affichent une vive aversion contre les modèles littéraires et autres codes théâtraux de l'Occident. Leur initiative de rupture ou de transgression s'inscrit inéluctablement dans une perspective d'affirmation de soi, de leur génie créateur, de leur identité, de leur originalité, voire de leur africanité. Il s'agit, pour eux, de vivre pleinement leur liberté de citoyens et de créateurs libres, de s'affirmer pleinement et de laisser libre cours à leur inventivité. Dans leur initiative anticonformiste, ces dramaturges africains de la deuxième génération semblent épouser les idéaux du « groupe de Coppet » ${ }^{1}$ dirigé par Madame de Staël. Les idéaux de ce groupe, fondés sur la nécessité de renouveler la dramaturgie française, 
stipulent que «chaque nation développe ses propres critères de goût, chaque époque crée son esthétique » (Bertrand, Haquette et al. 351).

Le souci de renouveler l'écriture théâtrale africaine pousse les dramaturges de la deuxième génération à discuter, à contester, à transgresser et à abandonner définitivement la tradition théâtrale occidentale et à inventer des formes dramatiques toutes neuves et affranchies de toutes conventions établies. Ils entendent, dans l'exercice de leur art et dans l'expression de leur pensée, faire absolument fi de tous les principes dramaturgiques existant au profit d'importantes recherches formelles puisées dans les profondeurs abyssales de leur créativité. Leurs écritures transgressives et révolutionnaires deviennent ainsi le reflet d'importantes innovations dramaturgiques, relevant de l'expérimentation de formes d'expression authentiques ancrées dans le culturel africain. Il s'agit en vérité de l'invention d'écritures dramatiques différentes, novatrices et libres qui consacrent avec pertinence non seulement la maturité et l'autonomie de la littérature négro-africaine mais aussi la capacité des dramaturges négro-africains à prendre de l'initiative, à inventer suivant des références non pas importées mais puisées de leur patrimoine culturel propre.

L'époque de la deuxième génération de dramaturges africains est aussi celle des grandes révolutions. C'est pourquoi les bouleversements d'ordre politique, social et même religieux ne cessent d'affermir davantage la notion de relativisme littéraire et esthétique. Les nouvelles écritures africaines ne sont pas en rupture seulement avec les canons dramaturgiques de l'Occident mais également avec ceux qui constituent la poétique théâtrale de la première génération africaine. En effet, la distance que prennent les dramaturgies africaines de cette nouvelle génération par rapport à la dramaturgie africaine antérieure est bien semblable à celle observée entre le Classicisme et le Romantisme. Alors que le Romantisme est présenté comme «l'art de présenter aux peuples les œuvres d'art qui, dans l'état actuel de leurs habitudes et de leurs croyances, sont susceptibles de leur donner le plus de plaisir possible », le classicisme est, quant à lui, présenté comme « la littérature qui donnait le plus grand plaisir possible à leurs arrière-grands-pères » (Liourré 66). Dans ce même ordre d'idées, la dramaturgie de la nouvelle génération est la manifestation de la liberté des peuples noirs et de leur authenticité pendant que celle de la première génération reste le signe de leur aliénation, de leur dépendance et de leur contrôle intellectuel par l'Occident. Ainsi, la dramaturgie africaine antérieure est considérée comme celle qui perpétue l'aliénation culturelle des créateurs africains pendant que les nouvelles dramaturgies, toutes novatrices, prennent, quant à elles, le parti de la liberté et d'affirmation de la personnalité africaine. 
Cette initiative dramaturgique d'affirmation de soi, de son identité, notamment l'identité africaine, prend l'appellation chez Victor Hugo de « libéralisme en littérature » (Liourré 51).

Ce faisant, les dramaturges, pour vivre pleinement leur liberté créatrice, choisissent d'adapter leurs compositions théâtrales à la vérité africaine que chacun d'eux entend sortir du silence de l'histoire et, de ce fait, "changent de moule autant de fois que de compositions » (Bertrand, Haquette et al. 358). Ils suivent, adoptent et appliquent à la lettre certains idéaux de la dramaturgie romantique développés par son chef de fil, Victor Hugo, à savoir, «Mettons le marteau dans les théories, les poétiques et les systèmes. Jetons bas ce vieux plâtrage qui masque la façade de l'art! Il n'y a ni règles, ni modèles; ou plutôt il n'y a d'autres règles que les lois générales de la nature qui planent sur l'art tout entier, et les lois spéciales qui, pour chaque composition, résultent des conditions d'existence propres à chaque sujet » (Bertrand, Haquette et al. 358). En effet, en littérature, la classification générique repose sur des normes fondamentales qui organisent de façon spécifique la construction de chacune des « chapelles » littéraires sur le plan formel. Pour ce qui est du domaine théâtral, «Episodes », «Actes » et « Tableaux » ont toujours constitué, au fil des siècles, les grandes articulations de la structure externe et s'affirment comme les «formes canoniques» des compositions dramaturgiques. Caractérisant la tragédie grecque, « les épisodes sont les parties dialoguées entre le prologue et l'exode (la sortie du chœur), ils se composent de longues tirades ou de stichomythies. Les épisodes pouvaient aller de deux (2) à six (6) » (Pavis 145). L'Acte, quant à lui, est indissociable de l'histoire du théâtre occidental car «il est une subdivision externe de la pièce en parties d'importance sensiblement égale en fonction du temps et du déroulement de l'action. L'Acte se définit comme une unité temporelle et narrative, en fonction de ses limites que par ses contenus » (25-26). Si la dramaturgie en Actes a évolué de trois à cinq, des auteurs latins et des théoriciens de la Renaissance à la tragédie classique, son hégémonie devient l'objet de contestation et de transgression à partir du dix-huitième siècle par la dramaturgie en Tableaux présentée comme suit : «L'apparition du tableau est liée à celle des éléments épiques dans le drame : le dramaturge ne focalise pas sur une crise, il décompose une durée, propose des fragments d'un temps discontinu. Il ne s'intéresse pas au lent développement, mais aux ruptures de l'action [...]. Au lieu du mouvement dramatique, il choisit la fixation photographique d'une scène » (382). En effet, les grandes unités dramatiques de découpage du texte auxquelles la tradition théâtrale nous a toujours habitué sont ici purement et simplement abandonnées et remplacées par des structures complètement nouvelles. Les dramaturges africains 
font, ainsi, de la licence inventive leur mode de rénovation de l'écriture dramatique africaine. Aussi, leur inventivité repose-t-elle sur la création de nouveaux éléments constitutifs du texte dramatique.

Dans L'œil de Bottey Zadi Zaourou, les éléments constitutifs de la structure externe organisant l'action dramatique ne sont que des astérisques disposés en forme de triangle équilatéral et employé dix-huit fois aux pages 71, 78, 86, 92, 95, 99, 102, 105, 109, 111, 114, 116, 117, 118, 121. Il s'agit là d'une structure externe particulièrement originale en raison justement des grandes unités dramatiques de découpage qui la composent. La majorité des pièces zadiennes sont, pour la plupart, imprégnées du patrimoine culturel négro-africain si bien qu'à propos de ses pièces, $L a$ guerre des femmes et La termitière, il affirme que la première est « le résultat d'une fusion de mythes et de légendes » (7) et la seconde " comporte évidemment quelques signes à forte connotation culturelle. De tels passages pourraient gêner les non-initiés à la culture africaine dans leur lecture du spectacle » (78).

Quant à Sony Labou Tansi, il a choisi de structurer sa pièce, La parenthèse de sang, avec des éléments relevant de l'ordre du temps ou les moments du jour, notamment le matin et le soir. Il s'agit des grandes unités dramatiques de structuration telles que « le premier soir » (5), « fin du premier soir » (29), «le deuxième soir » (30), « fin du deuxième soir » (43), «le troisième soir » (44), « fin du troisième soir » (55), « le quatrième soir » (56), « fin du quatrième » soir (66), « le premier matin » (67) qui sont précédées chacune d'un prologue. Ces éléments de structuration dramatiques donnent l'impression que l'action dramatique ne se déroule que le soir pour ne s'achever que le matin. Ces dramaturges, en l'occurrence Zaourou et Labou Tansi, ont, par leurs écritures dramatiques toutes subversives et originales, fait preuve d'une remarquable créativité qui consacre leur libération et leur liberté. Leurs esthétiques dramatiques deviennent le signe manifeste de leur identité, leur dignité, leur responsabilité de créateur pleinement assumée mais aussi leur liberté car «écrire, [...], c'est assumer une responsabilité et cette responsabilité désigne une liberté » (Barthes 10).

Outre les recherches formelles relevant de leur singulière inventivité, les dramaturges dits de la deuxième génération ont des rapports à la langue française particulièrement complexes. Ils se sont inscrits dans un processus d'adaptation et de réappropriation de celle-ci afin qu'elle cesse d'être un objet d'emprunt pour devenir un outil de communication authentiquement africain qui dit la réalité tel qu'elle est dite en Afrique. En effet, objet d'attention particulière marquée par une 
obsession de la correction grammaticale et syntaxique chez les premiers dramaturges africains, le français devient, chez ceux de la deuxième génération, un véritable champ d'expérimentation. Dans leur processus de récupération ou d'adaptation de cette langue étrangère et surtout coloniale, ils procèdent par l'ébranlement de ses formes stéréotypées et sa rigidité en générant de nouvelles façons d'exprimer la réalité dans les sociétés situées autour du cercle des tropiques, avec pour corollaire un métissage des formes et des structures littéraires et linguistiques.

Refusant d'être des «prisonnier[s] de la langue de l'autre » (Blachère 107), allusion faite au français, ils font passer cette langue dans un moule de modulation et de modélisation pour en faire un moyen de communication d'essence africaine semblable aux langues maternelles africaines que le colon a, à dessein, désagrégées et précipitées dans le monde du néant. Avec leurs travaux, le français subit une sorte de métamorphose, c'est-à-dire une dénaturation, pour devenir une langue « africanisée » ou une langue tout autre qui n’a rien à voir avec le français de France. À ce propos, Labou Tansi écrit : « nous sommes les locataires de la langue française. Nous payons régulièrement notre loyer. Mieux même : nous contribuons aux travaux d'aménagement dans la baraque. Nous sommes en partance pour une aventure de copropriation » (Zalesski 4). Une langue est une institution sociale et, en tant que tel, elle constitue un élément culturel fondamental permettant aux peuples qui l'ont en partage de se définir, s'identifier et s'affirmer pleinement. Elle est une donnée spécifique de l'identité culturelle, mieux même, elle possède « une identité spécifique liée aux caractères les plus intimes d'un peuple, à la nature de sa pensée et de son patrimoine, à sa perception des choses et sa façon de les considérer. C'est [elle] qui distingue les peuples les uns des autres » (Sow 22). Les peuples africains ne peuvent se définir, s'identifier et s'affirmer pleinement dans une langue qui leur est totalement étrangère; d'où la nécessité du travail consistant à articuler la langue française aux langues africaines pour l'adapter aux façons africaines d'exprimer la réalité.

Ce processus d'adaptation du français, voire de sa «tropicalisation » ou de son «africanisation », consiste en réalité en sa nationalisation par les dramaturges suivant leurs langues nationales. En effet, l'on découvre chez Labou Tansi ce qu'il convient d'appeler la « congolisation » du français, une autre forme de la licence créatrice, qui prend la forme et le sens d'une vraie provocation car la liberté textuelle se manifeste comme un acte délibéré de subversion du code de la langue française. En prenant appui sur la contestation du français de France et sur les revendications nationalistes et anticolonialistes, la tentative de «négrification » du français 
qu'affectionne tant Labou Tansi favorise l'émergence d'une écriture dramatique qui s'efforce d'exprimer dans toute son authenticité la sensibilité africaine. Chez lui, il s'agit moins de créer et d'inventer la réalité que de l'énoncer car, pour lui, «nommer une chose, c'[est] bien sûr la faire naître au monde des hommes, l'insérer dans l'ordre symbolique sans lequel son existence n'a aucune pertinence, mais c'[est] aussi et surtout la rendre animée en la dévoilant »(Devésa 102). Il a plié le français à l'expressivité africaine car, pour lui, « il n'était pas question de cracher de beaux mots de France après avoir respiré la foudre. Non. Il fallait dire ce qu'on dit chez nous avec une bouche de chez nous, penser ce qu'on pense chez nous tel qu'on le pense chez nous, voir ce qu'on voit chez nous avec des yeux de chez nous » (Devésa 163). À l'instar de Labou Tansi, Zaourou «ivoirise » le français de France en l'adaptant aux façons de dire la réalité dans la société ivoirienne dans tous ses démembrements, la rue y comprise.

Il s’agit en vérité d'une sorte de défrancisation du français de France qui consiste en des formes linguistiques nouvelles ou de nouveaux procédés de style censés rendre toute la profondeur de l'expressivité africaine et qui constituent pourtant des écarts linguistiques, donc des fautes, d'une inimaginable énormité par rapport à la norme linguistique française. En témoignent ces propos de l'homme-à-la-Tignasse : « alors, tout de suite, j'ai pris mon pieds la route, comme on dit chez nous, et je suis venu »(La tignasse, 69). Cet extrait permet de mieux percevoir la « défrancisation » du français de France dans les milieux sociaux ivoiriens :

Le joueur : « hôôô !!! fous-moi la paix oui ! Est-ce que c'est journal ça ? La chose où on parle de grands types seulement là ? »

Deuxième joueur : «Hé ! vous là ! si vous veu bagarre, il faut quitter ici hein ! Moi je sors la prison comme ça et pi mon l'affaire n'a même pas terminé encore » (10). Le marchand : « (montrant du doigt un panneau) Ti na pa vi ça là ? Ya papelace ici ! »;

L'inconnu : «Tu ne vois pas que type-là est fou ? laissé luiii ! Esqu'y a pas l'autre vendère là-bas ? hôôôô ! Toi aussi » (38).

Le marchand : "Qui lé fou ? hein ? Qui lé fou ? C'est vous-mêmes qu'on est fou woui ! Fouti-moi le camp ici ! vous n’a pas vi praque là ? y a pas pelace ici. Hallez ! Fouti-moi la paix»

Le marchand : «Qui ce kia vous fait ici même ? Vous n'a pas vi praque là ? On dit vous quéya pas pilace épi vous lé là ka même. Vraiment... Vous-même... C'est pas la peine quoi. (Vivement) C'est pou ça ké nous onpézamais fait kékéchose là. Ôôô̂ô !... » (39).

Il s'agit là d'un français populaire auquel s'ajoute le parler de la rue. Il est clair que les habitants de la rue des villes africaines parlent le français mais un français qui est encore différent du français populaire. Si celui-ci constitue une entorse au français de France, le français de la rue est un 
français tout autre qui a ses propres normes syntaxiques et sa propre logique. C'est en fait le signe évident que chaque milieu social possède son langage, c'est-à-dire son code langagier propre.

\section{Conclusion}

Au terme de cette réflexion sur l'expression de liberté dans les dramaturgies africaines postcoloniales, il convient de retenir que les dramaturges africains de la deuxième génération, en l'occurrence Zaourou et Labou Tansi, ont, plus que leurs prédécesseurs de la première génération, accouché d'esthétiques dramaturgiques originales révélant l'identité et la personnalité africaines. Aussi ont-ils soumis le français, langue étrangère et surtout coloniale, à un processus d'adaptation et de réappropriation par son articulation à l'expressivité authentiquement africaine. Ils ont ainsi créé sur la scène africaine un théâtre avec une spécificité à la fois esthétique et linguistique. En somme, par ce travail novateur, le théâtre africain d'expression française devient désormais théâtre d'expression africaine.

\section{Bibliographie}

Adiaffi, Jean-Marie. «Les maîtres de la parole ». Magazine littéraire 195 (1983).

Barthes, Roland. Le plaisir du texte. Paris : Seuil, 1973.

Blachère, Jean-Claude. Négritudes. Les écrivains d'Afrique noire et la langue française. Paris : Harmattan, 1993.

Bertrand, Dominique, Jean-Louis Haquette, Laurence Hélix et al.. Le théâtre : premier et second cycles universitaires. Rosny : Bréal. coll. « Grand amphi : littérature », 1996.

Devésa, Jean-Michel. Sony Labou Tansi : écrivain de la honte et des rives magiques du Kongo. Paris : Harmattan, 1996.

Goldman, Lucien. Pour une sociologie du roman. Paris : Gallimard, 1964.

Kotchy, Barthélemy. «Discours inaugural ». Actes du colloque sur le théâtre négro-africain. École des Lettres et Sciences humaines, 15-29 avril 1970. Paris : Présence Africaine, 1971. 9-12.

Kotchy, Barthélemy et Kesteloot, Lilyan. Aimé Césaire : l’homme et l'œuvre. Paris : Présence Africaine, 1993.

Kourouma, Ahmadou. Les soleils des indépendances. Paris : Seuil, 1968.

Labou Tansi, Sony. La parenthèse de sang suivi de Je soussigné cardiaque. Paris: Hatier, 
1981.

Lavergne, Evelyne. «Les soleils des indépendances : un roman authentiquement africain ». Revue de Littérature et d'Esthétique Négro-africaine 3. Abidjan : Nouvelles Éditions Africaines, $1981: 15-25$.

Liourré, Michel. Le Drame. Paris : Colin, 1963.

Makward, Idriss. « Sony Labou Tansi et le 'français' du Congo ». Sony Labou Tansi : témoin de son temps. Dir. N’da Pierre Lézou Gerard. Limoges : PUBLIM, 2003 : 29-38.

Pavis, Patrice. Dictionnaire du théâtre. Paris : Messidor/Sociales, 1987.

Sido, Issa. «La politique ». Actes du colloque sur le théâtre négro-africain. École des Lettres et Sciences humaines, 15-29 avril 1970. Paris : Présence Africaine, 1971 : 94-99.

Sow, Alpha Issouf. « Prolégomènes ». Introduction à la culture africaine. Paris : Unesco / Union Générale d'Éditions, 1977 : 9-46.

Traoré, Bakary. «Le théâtre africain : réalités et perspectives ». Actes du colloque sur le théâtre négro-africain. École des Lettres et Sciences humaines, 15-29 avril 1970, Paris : Présence Africaine, 1971 : 54-61.

---. « Le théâtre africain de l'École William-Ponty ». Actes du colloque sur le théâtre négroafricain. École des Lettres et Sciences humaines, 15-29 avril 1970. Paris : Présence Africaine, $1971: 37-44$.

Zalesski, Michèle. «Locataires de la même maison ». Entretien avec Sony Labou Tansi. Diagonales 9 (1989) : 3-4.

Zaourou, Bottey Zadi. Les sofas suivi de L'œil. Paris : Harmattan, 1983.

---. La tignasse. Abidjan : CEDA, 1984.

---. La guerre des femmes suivie de La termitière. Abidjan : NEI, 2001.

\footnotetext{
Note

${ }^{1}$ Coppet est une commune suisse où Germaine de Staël détenait une propriété d'où elle réunissait autour d'elle des Français, des Allemands, des Suisses pour des échanges qui donnèrent forme au premier romantisme français.
} 